\title{
4.5 PULSARS AND CLOSE BINARY SYSTEMS
}

\author{
VIRGINIA TRIMBLE and MARTIN REES \\ Institute of Theoretical Astronomy, Cambridge, U.K.
}

\begin{abstract}
It is first considered what must happen if pulsars (i.e. neutron stars) are formed in close binary systems (CBS), and whether the resulting orbital motion and mass transfer should be observable. As this set of alternatives seems unlikely, there follow suggestions of how one might prevent the formation of neutron stars in close binaries. Finally, it is shown that 'runaway' pulsars with velocities larger than about $15 \mathrm{~km} / \mathrm{sec}$ cannot be produced by isotropic supernova explosions within close binaries, and an alternative explanation is suggested for the observed correlation of periods of pulsars with their distances from the galactic plane.
\end{abstract}

\section{Introduction}

It is known (with decreasing levels of certainty) that: (1) an appreciable fraction of all stars occur in close binary systems (CBS). (2) some stars end their lives in supernova events, (3) some supernovae produce neutron stars, and (4) some neutron stars are observed as pulsars. At least a few pulsars might, therefore, be expected to occur in close binary systems.

\section{Pulsars in Close Binary Systems}

Two detectable effects should occur for pulsars which have close companions - orbital motion and accretion of material. Periodic changes in pulse arrival times with the amplitudes and periods expected from binary motion have not been observed for any pulsar. For the 15 of 50 known objects for which rates of change of period have been detected (Terzian, 1970), changes in orbital velocities of more than about $3 \times 10^{-8}$ the velocity of light per 100 days can be excluded. This corresponds to separations greater than $40 \sqrt{ }\left(M_{1}+M_{2}\right) \mathrm{AU}$, which is so large that the systems cannot be considered close, in either the intuitive sense or in the sense that mass exchange will occur as the component stars evolve. Corrected for projection effects and the statistics of small numbers, the available data show that not more than one pulsar in four or five can belong to a close binary system. Additional observations of period changes must either reveal binary motion or lower this limit considerably.

Consider now what must happen in a CBS where the less massive component is a neutron star. (Mass exchange in earlier phases of the history of the system will probably prevent the neutron star ever being the more massive component; Plavec, 1968a, b.) In due course, the ordinary star will evolve, increase its radius, and, if the system is sufficiently close, fill its Roche lobe. Matter is then free to flow toward the neutron star, which may or may not be a pulsar at the time. At least some of the matter will probably be captured and retained by the neutron star. This is the case for a white dwarf under similar circumstances (Giannone and Weigert, 1967).

There is, however, an upper limit to the rate at which matter can be accumulated 
on the neutron star. For any object radiation pressure more than balances gravity if $L / M \geqslant 6 \times 10^{4}$ in solar units. Since material falling onto a neutron star must get rid of about $10^{20} \mathrm{ergs} / \mathrm{gram}$ of gravitational potential energy, matter cannot be added faster than about $2 \times 10^{18} \mathrm{~g} / \mathrm{sec}$ or $3 \times 10^{-8} M_{\odot} / \mathrm{yr}$. If the material attempts to fall straight down, anything in excess of this limit will be blown off by radiation pressure. In the case of the white dwarf, the primary energy source is nuclear reactions rather than gravitational potential energy, and the corresponding limit is about $3 \times 10^{19} \mathrm{~g} / \mathrm{sec}$ or $5 \times 10^{-7} M_{\odot} / y r$. The resulting system, alternately transferring and blowing off material, might look not unlike $T$ Coronae Borealis, the only recurrent nova known to occur in a wide binary.

The real situation is probably not this simple. Material transferred in a CBS will carry considerable angular momentum (unlike, for instance, matter accreted by a neutron star from the interstellar medium, as discussed by Ostriker et al., 1970). It will, therefore, not fall straight in, but form a disc around the neutron star, dropping down only as fast as viscosity transfers angular momentum from the inner to the outer parts of the disc. If viscosity were sufficiently small, the disc might persist much longer than mass transfer lasted (typically the dynamical or thermal time scale rather than the nuclear time scale) and all the matter eventually be captured. If only molecular viscosity counted, this would probably be the situation.

The relevant time scale, $\tau$, is of the order $4 \times 10^{-11} R^{2} N T^{-5 / 2}$ where $R$ is the radius of the disc, $N$ its average (particle) density, and $T$ its temperature. For a solar mass or so of material filling the Roche lobe around the neutron star in a CBS at a temperature of $10^{7} \mathrm{~K}, \tau$ is about $3 \times 10^{9} \mathrm{y}$. Including turbulent and magnetic viscosity will decrease $\tau$ very considerably, and it is probable that material will fall in as fast as the $L / M$ limits will let it. There is one further process to be considered. The $10^{20} \mathrm{erg} / \mathrm{g}$ available from gravitational potential energy would suffice to raise the temperature of the infalling material to nearly $10^{12} \mathrm{~K}$. At such exalted temperatures, the crosssections for processes producing neutrinos are no longer negligible. And, since any neutrinos formed will pass freely through the disc, energy radiated in this form is exempt from the $L / M$ restriction. Thus a mechanism is perhaps available that will allow mass to be accepted by a neutron star as fast as its companion wants to get rid of that mass, even without storing it in a disc.

If sufficient mass deposition occurs to raise the neutron star above the largest stable mass for such a configuration, then either some violent event will take place (which may be presumed to disrupt the system) or the star will collapse to a black hole. Systems undergoing mass transfer and/or deposition should be short period (less than $10 \mathrm{yr}$ or so) spectroscopic binaries showing lines of at most one star (and, perhaps, a disc), in which the invisible component is more massive than the Chandrasekhar limit. Tables of the 50 singleline spectroscopic binaries with well-determined orbits containing an unseen star larger than $1.4 M_{\odot}$ are given by Trimble and Thorne (1969). None of these systems has a primary component which completely fills its Roche lobe, nor can any of them have matter falling from a disc to the neutron star at more than $10^{-3}$ of the limiting rate, unless neutrino energy loss is extremely efficient. 
Matter falling on a neutron star should release its gravitational potential energy largely in the form of X-rays (Shklovsky, 1967, and others). None of the tabulated systems coincides with any X-ray source position in the catalogues of Dolan (1970), Matsuoka (1970), or Seward (1970). We can thus set limits on their X-ray luminosities. Consider system No. 27 of Trimble and Thorne's Table 1. This system is far enough from the galactic plane that X-ray source confusion is not a problem, and its distance (which is typical for these systems) can be determined fairly accurately. The primary is a Cepheid variable of period $8.38\left(M_{V} \sim-3.7\right)$ seen at apparent magnitude 5.87 to 7.02. The system is, therefore, about $1 \mathrm{kpc}$ from us. Its $X$-ray flux at the earth is surely not greater than $5 \times 10^{-9} \mathrm{erg} \mathrm{cm}^{-2} \mathrm{sec}^{-1}$ in the 1.5 to $5 \mathrm{keV}$ band. (Weak observed sources are typically around $2 \times 10^{-9} \mathrm{erg} \mathrm{cm}^{-2} \mathrm{sec}^{-1}$ in this energy interval.) Thus less than $10^{35} \mathrm{ergs} / \mathrm{sec}$ are being radiated as soft X-rays - or as any sort of X-rays unless the spectrum is very flat. This is less than $10^{-3}$ of the limiting $L / M$ rate which we expect when radiation pressure rather than mass transfer rate or viscosity limits the mass deposition. Thus, in all of the tabulated systems, either no disc has been formed or all but $10^{-3}$ of the energy of accreting matter is being radiated in some form other than X-rays - presumably neutrinos. This degree of efficiency seems improbable.

The above estimate is a generous one; the secondaries having $M \gtrsim 2.0 M_{\odot}$ cannot be neutron stars, and matter falling onto a black hole may radiate more than $10^{20}$ erg/gram in some form or other.

Eclipsing systems have been excluded from consideration. One might imagine material lost by a primary forming a rapidly rotating disc around a black hole or neutron star which was sufficiently substantial to produce eclipses. Such a system would look rather like $\beta$ Lyrae (which is probably still in a phase of rapid mass transfer) and the secondary component (in the absence of X-ray emission produced as the disc material falls in) could not be distinguished from an ordinary star surrounded by a similar disc. There are again no significant position coincidences of observed X-ray sources with the single-line eclipsing systems tabulated by Trimble and Thorne (1969).

More generally, there is a limit to the input rate of soft X-rays into the Galaxy from the temperatures of interstellar $\mathrm{HI}$ clouds. It is about $10^{40} \mathrm{erg} / \mathrm{sec}$. The contribution from all neutron stars accreting matter (in or out of CBS's) must not exceed this. Neutron stars passing through the general interstellar medium may supply a significant fraction of this (Ostriker et al., 1970). In any case, if $L / M=6 \times 10^{4}$ and $M$ is about one solar mass, at most 40 objects can be fitted in under the upper limit.

Thus, if one pulsar is formed every $30 \mathrm{yr}$ (Gunn and Ostriker, 1970), the time during which matter is accreted multiplied by the fraction of pulsars in close binaries is at most $1200 \mathrm{yr}$. If infalling matter is partially blown off by radiation pressure and/or neutrino losses are important, the accretion time will be no greater than the time during which the primary wants to lose mass. This is typically a thermal time scale and is in the range $10^{4}$ to $10^{6} \mathrm{yr}$. Thus at most 0.1 to 0.001 of pulsars formed in the recent past can have remained in CBS. If neutron stars which are not pulsars occur 
in close binaries these limits must be made more stringent. The formation of a disc in which viscosity is so low that matter continues to fall in at the limiting rate of $2 \times 10^{18} \mathrm{~g} / \mathrm{sec}$ until all the disc material has radiated away its potential energy (this takes $10^{8}$ years or so) would lower the limit to one pulsar in $10^{5}$ in a binary system.

One might get around the X-ray limit by postulating, ad hoc, a large (e.g. $R \sim 2 \times 10^{15} \mathrm{~cm}$ at $T=600 \mathrm{~K}$ ) cloud around each such system which transforms it into an infra-red source (and incidentally accounts for the rarity of optical identifications of pulsars). Because the galactic infrared background is stronger than the $X$-ray background most pulsars formed might radiate at the limiting rate for $10^{5} \mathrm{y}$ or so. The binary systems discussed above could not, of course, have their X-rays hidden in this fashion because we see the objects optically.

The conclusion of Trimble and Thorne (1969) that we probably do not observe any binary systems containing neutron stars or black holes is thus reiterated. Nor can many of the pulsars formed in the past be members of CBS's without violating X-ray limits. The evidence of pulsar period constancy suggests that the vast majority of such objects now observed do not have close companions.

\section{Pulsars not in Close Binary Systems}

Evidently one of two things must happen - either most stars in CBS's do not undergo the sort of supernova event which produces pulsars or the explosion always disrupts the system - or both. It would not be surprising if binary components were to end their lives in some rather different fashion from single stars. The initial primary in a CBS, which always remains the more rapidly evolving star (Paczynski, 1967) although it becomes the less massive after mass exchange, will have lost much or all of its hydrogen-rich envelope before reaching the supernova stage. This may actually influence the course of interior evolution, for instance by preventing the explosive carbon ignition (Paczynski, private communication) which perhaps produces some supernovae, and it may well be critical in determining how much matter is expelled in the event, how much implodes, and how far. If Arnett's (1971) explanation of supernova light curves is correct, the loss of the hydrogen envelope will drastically change what we see of the event.

Alternatively, the system may be disrupted. Gunn and Ostriker (1970) have suggested that this usually happens, both the neutron star and the secondary flying off as runaways. This provides an explanation of the observed correlation of periods of pulsars with their distances from the galactic plane and of the supposedly anomalous proper motion of the Crab Nebula (but see elsewhere in this volume). The systems are presumed to start with a scale height of about $80 \mathrm{pc}$ and the neutron stars to fly off with a Maxwellian distribution of velocities peaked at $100 \mathrm{~km} / \mathrm{sec}$.

Unfortunately, the phenomenon of mass exchange has not been taken into account in formulating this picture. In order for an isotropic expansion to unbind the system, it is necessary that the difference of the component masses, $M_{1}-M_{2}$, be greater than $2 M_{N 1}$ (twice the mass of the neutron star formed). It is clear that, once mass transfer 
has transformed the initial primary into the less massive (but still more rapidly evolving) star, no possible explosion of this star, $M_{1}$, can fulfill the condition. It is, therefore, necessary that the star not fill its Roche lobe at any phase preceding supernova explosion.

This restriction allows upper limits to be placed on runaway velocities from systems of various masses, by requiring that the more massive star not be larger than the radius of its Roche lobe at any phase of stellar evolution which has been studied. Formulae given by Gott et al. (1970) can be combined to yield the velocity of the neutron star after the explosion,

$$
V_{N 1}=\frac{G^{1 / 2} M_{2}\left(M_{1}-M_{N 1}\right)}{\mathbf{a}^{1 / 2}\left(M_{1}+M_{2}\right)^{1 / 2}\left[M_{N 1}+\left\{M_{1}^{2}-2 M_{N 1}\left(M_{1}+M_{2}\right)\right\}^{1 / 2}\right]}
$$

where $\mathbf{a}$ is the semi-major axis of the binary orbit, $\mathrm{G}$ the gravitational constant, and the $M$ 's as above. Radii of Roche lobes in terms of a are given by Kopal (1959), and maximum radii reached by stars of various masses can be taken from Paczynski (1970).

TABLE I

Upper limits to runaway velocities of neutron stars formed by supernovae in close binary systems

\begin{tabular}{lllllll}
\hline $\begin{array}{l}M_{1} \\
\text { (solar masses) }\end{array}$ & $\begin{array}{l}M_{2} \\
\text { (solar masses) }\end{array}$ & $\begin{array}{l}M_{N 1} \\
\text { (solar masses) }\end{array}$ & $R_{1} \max (\mathrm{cm})$ & $R^{10 b \mathrm{e}} / a$ & $a^{\min }(\mathrm{cm})$ & $\begin{array}{l}V_{N 1} \max \\
(\mathrm{km} / \mathrm{sec})\end{array}$ \\
3 & - & & & & \\
3 & $\frac{1}{2}$ & $\frac{1}{2}$ & $7.59 \times 10^{13}$ & 0.567 & $1.34 \times 10^{14}$ & 2.3 \\
3 & 1 & $\frac{1}{2}$ & $7.59 \times 10^{13}$ & 0.469 & $1.62 \times 10^{14}$ & 4.1 \\
3 & $\frac{1}{2}$ & 1 & $7.59 \times 10^{13}$ & 0.567 & $1.34 \times 10^{14}$ & 2.2 \\
5 & 1 & $\frac{1}{2}$ & $7.59 \times 10^{13}$ & 0.469 & $1.62 \times 10^{14}$ & 4.5 \\
5 & 2 & $\frac{1}{2}$ & $7.59 \times 10^{13}$ & 0.461 & $1.65 \times 10^{14}$ & 6.4 \\
5 & 4 & 1 & $7.59 \times 10^{13}$ & 0.398 & $1.91 \times 10^{14}$ & 11.1 \\
5 & 2 & 1 & $7.59 \times 10^{13}$ & 0.461 & $1.65 \times 10^{14}$ & 6.3 \\
7 & 4 & $\frac{1}{2}$ & $7.59 \times 10^{13}$ & 0.398 & $1.91 \times 10^{14}$ & 12.2 \\
7 & 4 & $\frac{1}{2}$ & $7.42 \times 10^{13}$ & 0.429 & $1.77 \times 10^{14}$ & 10.2 \\
7 & 6 & 1 & $7.42 \times 10^{13}$ & 0.398 & $1.19 \times 10^{14}$ & 13.9 \\
7 & 4 & 1 & $7.42 \times 10^{13}$ & 0.429 & $1.77 \times 10^{14}$ & 10.1 \\
& 6 & & $7.42 \times 10^{13}$ & 0.398 & $1.91 \times 10^{14}$ & 14.4
\end{tabular}

The resulting upper limits to $V_{N 1}$ are given in Table I. The primary masses have been chosen to be large enough that the stars will have a fairly small scale height in the Galaxy and small enough that the stars will be reasonably common objects. The secondary masses were chosen to give the largest possible runaway velocities consistent with the system becoming unbound.

None of these velocities is anywhere large enough to explain the observed periodscale height correlation, which must, therefore, be otherwise accounted for. Notice that ordinary runaway stars (once secondaries of systems with very massive - e.g. $50 M_{\odot}$ - primaries; Blaauw, 1961) cannot be the ancestors of pulsars. In order to produce an age-scale height correlation, the high velocity must be acquired at or near the time the pulsar is formed. If the object had been given a high velocity at a time 
before the supernova event comparable with pulsar ages $\left(10^{6}-10^{7} \mathrm{y}\right)$ then all objects would have the same scale height, independent of age (i.e., period).

A way of producing the required velocity is immediately suggested by the dynamics of the Crab Nebula - NP 0531 system. As reported elsewhere in this volume, the nebula and pulsar have proper motions differing by about $0.014 \pm 0.003^{\prime \prime} / \mathrm{yr}$. This, at a distance of $1500 \mathrm{pc}$, amounts to $100 \mathrm{~km} / \mathrm{sec}$, and it has not been suggested anywhere that the star which became SN 1054 was other than single at the time of the explosion.

The period-scale height relation thus finds ready explanation in recoil of the neutron star from an asymmetric supernova explosion. Such asymmetry would also, of course, allow the neutron star to be expelled with larger velocity from a binary system, but the companion star is in no way necessary for the phenomenon to occur.

If on the other hand supernova explosions are usually asymmetrical enough to give the resulting neutron star a 'kick' of $100 \mathrm{~km} / \mathrm{sec}$ then most of the objects starting out in binaries should be expelled.

The explanation suggested by Michel (1970) for high pulsar velocities also involves a close pair of stars - in this case both of them neutron stars, but as his binary systems have never passed through a main sequence phase (having been formed from a single star) mass transfer is irrelevant.

\section{Acknowledgements}

Many of the ideas in this discussion were contributed by Dr. B. Paczynski, who, however, assumes no responsibility for the authors' having made proper use of them. V. T. gratefully acknowledges a NATO Postdoctoral Research Fellowship.

\section{References}

Arnett, W. D.: 1971, Astrophys. J. 163, 11.

Blaauw, A.: 1961, Bull. Astron. Inst. Neth. 15, 265.

Dolan, J. F.: 1970, Astron. J. 75, 223.

Gianone, P. and Wiegert, A.: 1967, Z. Astrophys. 67, 41.

Gott, J. R., Gunn, J. E., and Ostriker, J. P.: 1970, Astrophys. J. 160, L91.

Gunn, J. E. and Ostriker, J. P.: 1970, Astrophys. J. 160, 979.

Kopal, Z.: 1959, Close Binary Systems, Chapman and Hill, London, p. 136.

Matsuoka, M.: 1970, Univ. of Tokyo, Space Aeronautics Sci. Report No. 445.

Michel, F. C.: 1970, Nature 228, 1072 and this symposium, Paper 6.8, p. 378.

Ostriker, J. P., Rees, M. J., and Silk, J.: 1970, Astrophys. Letters 6, 179.

Paczynski, B.: 1967, Acta Astron. 20, 47.

Plavec, M.: 1968a, Astrophys. Space Sci. 1, 239.

Plavec, M.: 1968b, Advances Astron. Astrophys. 6, 201.

Seward, F. D.: 1970, Univ. Calif. Livermore Preprint, UCID 15622.

Shklovsky, I. S.: 1967, Astrophys. J. 148, L1.

Terzian, Y.: 1970, privately circulated pulsar tables.

Trimble, V. L. and Thorne, K. S.: Astrophys. J. 156, 1013. 